DOI: 10.15593/RZhBiomeh/2015.4.02

УДК 531/534:[57+61]
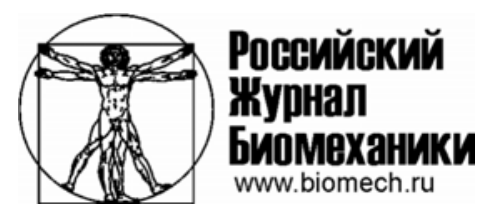

\title{
ЗАДАЧА КОРРЕКЦИИ ПРИКУСА В ЗУБОЧЕЛЮСТНОЙ СИСТЕМЕ ЧЕЛОВЕКА
}

\author{
В.М. Тверье, В.Н. Никитин
}

Кафедра теоретической механики и биомеханики Пермского национального исследовательского политехнического университета, Россия, 614990, Пермь, Комсомольский проспект, 29, e-mail: nikitinvladislav86@gmail.com

\begin{abstract}
Аннотация. При коррекции прикуса, связанной с потерей зубов, повышенной стираемостью зубов, травмами и переломами челюстей, стоматолог опирается на свой опыт или использует методики, в основе которых заложены геометрические расчеты. Прикус определяет нагружение всей нижней челюсти и височнонижнечелюстных суставов, поэтому необходимо биомеханическое сопровождение такой коррекции. Поставлена задача управления коррекцией прикуса, в которой при определяемых параметрах прикуса напряжения в дисках суставов и теле нижней челюсти не должны превышать заданных пределов для интенсивности напряжений. Напряжения и деформации в теле нижней челюсти и дисках определяются из решения двух задач теории упругости: для области нижней челюсти и области диска при центральной окклюзии при максимальном нагружении мышц. Точка приложения реакции сустава и положение диска в суставе определяются в нем по результатам магнитно-резонансной томографии. Для определения напряженно-деформированного состояния челюстей и диска височно-нижнечелюстного сустава необходимо знать усилия в мышцах и реакцию височно-нижнечелюстного сустава, являющихся граничными условиями для поставленных задач. Величины усилий мышц заведомо неизвестны. Усилия мышц определяются из решения задачи оптимизации, в которую входят уравнения статики нижней челюсти под действием заданной жевательной нагрузки и критерий оптимизации. После вычисления усилий мышц и реакций в височнонижнечелюстных суставах решается первоначальная задача определения напряженно-деформированного состояния нижней челюсти и диска. Из сравнения полученных напряжений с допустимыми вычисляются необходимые параметры, определяющие положение прикуса.
\end{abstract}

Ключевые слова: коррекция прикуса, зубочелюстная система, височнонижнечелюстной сустав, усилия мышц, суставной диск.

\section{ВВЕДЕНИЕ}

Изменения зубочелюстной системы связаны с процессами питания, дыхания, глотания и речи, но они влияют и на весь организм человека. Зачастую не строятся взаимосвязи между патологическими процессами, происходящими в зубочелюстной системе и других системах организма человека, которые, на первый взгляд, не имеют ярко выраженных причинных связей [7-13]. Анализ изменений зубочелюстной системы предполагает персонифицированный подход (patient specific) в рамках концепции виртуального физиологического человека (virtual physiological human).

\footnotetext{
(C) Тверье В.М., Никитин В.Н., 2015

Тверье Виктор Моисеевич, к.т.н., доцент кафедры теоретической механики и биомеханики, Пермь Никитин Владислав Николаевич, старший преподаватель кафедры теоретической механики и биомеханики, Пермь
} 
Основным из параметров, определяющих состояние зубочелюстной системы, является прикус (взаимное расположение верхней и нижней челюстей) (рис. 1). Предполагается проследить более подробно влияние изменений прикуса на состояние диска височнонижнечелюстного сустава и качественно оценить возможность биомеханического влияния изменений диска височно-нижнечелюстного сустава на мозговое кровоснабжение.

При коррекции прикуса зубочелюстной системы, связанной с потерей зубов, их повышенной стираемостью, травмами и переломами челюстей, стоматологи опираются на свой опыт и используют методики оценки взаимного положения верхней и нижней челюстей на основе геометрических расчетов, производимых в результате диагностики (рис. 2). Стоматолог, опираясь на диапазоны нормальных значений параметров, определенных в процессе диагностики [14], корректирует прикус, изменяя высоту и характер смыкания зубов верхней и нижней челюстей, применением различных ортопедических конструкций и ортодонтического лечения (см. рис. 1) [1, 14, 15, 18]. В ходе процедуры коррекции прикуса стоматолога устраивает случай попадания параметров, определяющих состояние прикуса, в диапазон нормальных значений [14]. Он выбирает ортопедическую конструкцию, руководствуясь своим опытом. Таким образом, при коррекции прикуса оцениваются только геометрические параметры его состояния, которые косвенно позволяют установить, какие усилия возникают в элементах зубочелюстной системы. При этом косвенно, на основании опыта стоматолога, оценивается, сможет ли применяемая ортопедическая конструкция нести нагрузку, которая возникает в ней у конкретного пациента.

Состояние прикуса определяет нагружение всей нижней челюсти и височнонижнечелюстного сустава, поэтому необходимо биомеханическое сопровождение такой коррекции. Для объективизации методики коррекции прикуса нужны количественные оценки состояния диска височно-нижнечелюстного сустава и костной ткани нижней челюсти. В качестве количественной оценки предлагается рассматривать величины усилий, возникающих в диске височно-нижнечелюстного сустава и в самой нижней челюсти. Для этого поставлена задача управления коррекцией прикуса, в которой при определяемых параметрах прикуса напряжения в диске сустава, мыщелке и теле нижней челюсти не должны превышать заданных пределов для интенсивности напряжений.

Большинство стоматологов не применяет каких-либо измерений и методов диагностики для оценки состояния зубочелюстной системы человека. В настоящее время зубочелюстная система рассматривается как специализированная, многоблочная, многофункциональная биомеханическая [11-13]. Применение современных

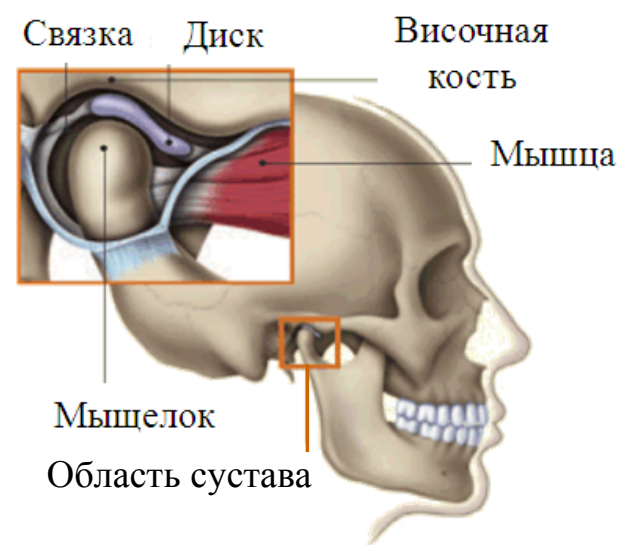

Рис. 1. Область височно-нижнечелюстного сустава 


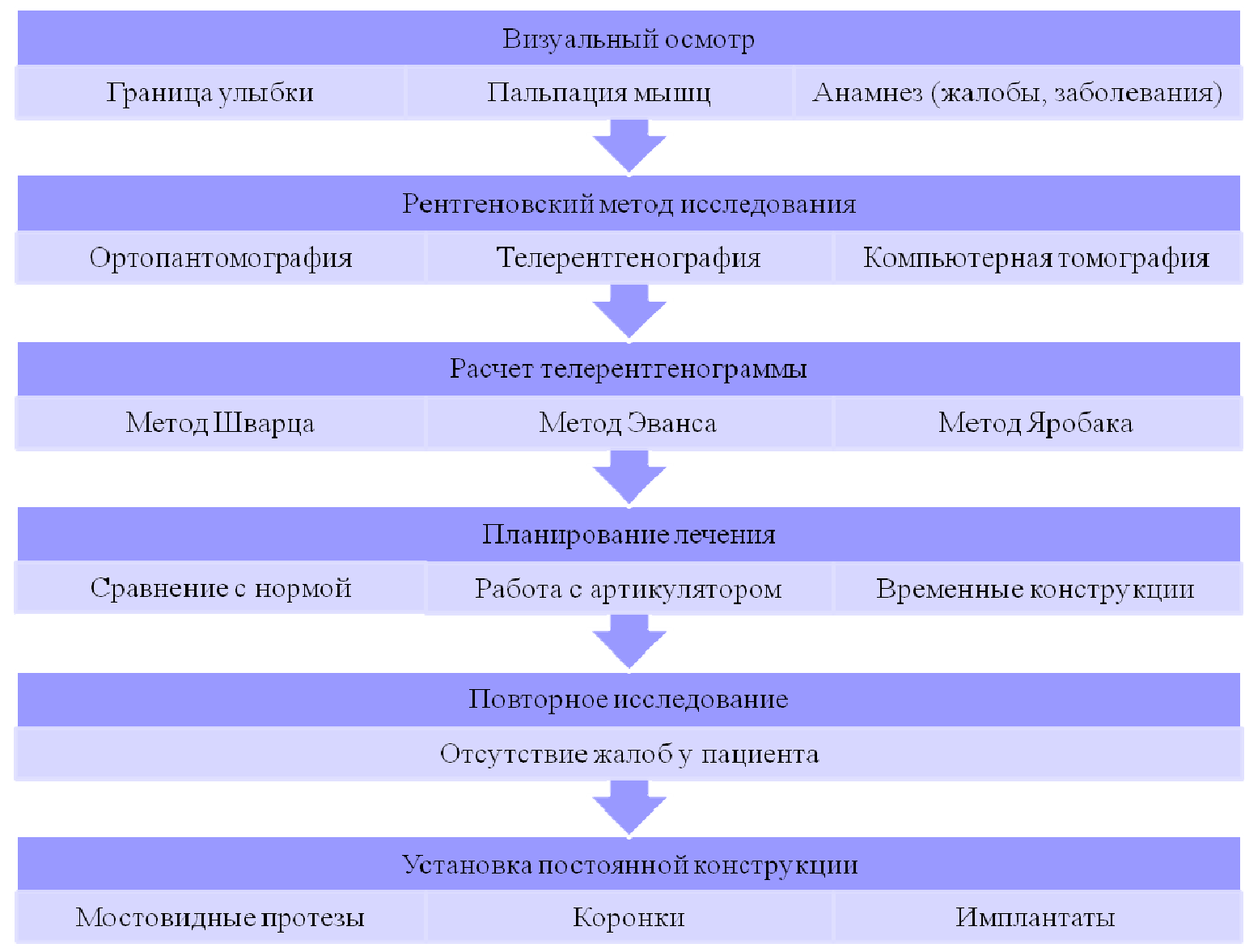

Рис. 2. Методика коррекции прикуса зубочелюстной системы [14]

методов диагностики состояния зубочелюстной системы не позволяет в полной мере оценить усилия, возникающие в ней. Ортопантомография (панорамный снимок), телерентгенография (фронтальный и боковой снимки), конусно-лучевая и компьютерная томографии (трехмерная визуализация) позволяют оценить геометрические соотношения только костных элементов зубочелюстной системы. Благодаря рентгеновским методам представляется возможным оценить геометрические размеры суставной щели височно-нижнечелюстного сустава между суставными поверхностями: суставным бугорком и ямкой височной кости и суставным мыщелком нижней челюсти.

Магнитно-резонансная томография применяется стоматологами реже по сравнению с вышеупомянутыми рентгеновскими методами по временным затратам. Она позволяет увидеть не только костные, но и мягкотканые структуры: мышцы, связки. А самое главное, магнитно-резонансная томография позволяет оценить положение диска височно-нижнечелюстного сустава относительно суставных поверхностей.

Для полного анализа состояния зубочелюстной системы недостаточно знания только геометрии ее структур. Необходимо знать усилия, возникающие во всей зубочелюстной системе, так как при превышении предельных значений появляются патологические изменения.

Для определения усилий в зубочелюстной системе необходимо поставить задачу определения напряженно-деформированного состояния нижней челюсти и диска височно-нижнечелюстного сустава под действием усилий жевательных мышц, приводящих в движение нижнюю челюсть. На данный момент не существует метода, позволяющего количественно оценить усилия каждой мышцы in vivo $[3,16,21,24,26]$. 


\section{ПАРАМЕТРЫ ЗУБОЧЕЛЮСТНОЙ СИСТЕМЫ, АНАЛИЗИРУЕМЫЕ ПРИ РАСШИФРОВКЕ ТЕЛЕРЕНТГЕНОГРАММЫ}

Телерентгенография - рентгеновский метод, основанный на получении фронтального и бокового снимков. Наибольший интерес представляет боковой снимок, благодаря которому можно оценить взаимоотношение верхней и нижней челюстей, положение и наклон зубов по отношению к челюстям, а также размеры суставной щели височно-нижнечелюстного сустава.

При анализе бокового снимка телерентгенограммы стоматолог отмечает ряд точек, отражающих геометрические аспекты зубочелюстной системы. При знакомстве с различными методиками расчета телерентгенограммы и подходами стоматологов (выделение на снимке тех или иных точек, определение линейных и угловых параметров) выяснилось, что каждый стоматолог выбирает удобный для себя метод и важные и необходимые для него параметры для коррекции прикуса.

При анализе телерентгенограмм основными показателями, характеризующими геометрию и состояние зубочелюстной системы, являются углы. В литературе описано, что линейные размеры челюстей и расстояния между специальными точками сильно варьируются, а угловые параметры имеют меньшие пределы, и для них в течение многолетних исследований установлена норма.

В ходе анализа телерентгенограмм были выделены три независимых параметра (угла), отвечающие за положение нижней челюсти относительно верхней (черепа):

1. Угол $A N B$ - угол, отвечающий за взаимное расположение челюстей (перекрывание челюстей) и характеризующий, на сколько нижняя челюсть позади или впереди верхней челюсти (в норме $2 \pm 2^{\circ}$ ) (рис. 3).

2. Угол $F H-M L-$ угол, отвечающий за наклон нижней челюсти по отношению к верхней (черепу) (в норме $24 \pm 3^{\circ}$ ) (рис. 4).

3. Угол $D o A-$ угол, отвечающий за наклон нижней челюсти по отношению к верхней (черепу) (в норме $43 \pm 2^{\circ}$ ) (рис. 5). На рис. 6 показан способ определения положения точки $X i$.

Проанализируем, как координаты точек влияют на изменение вышеуказанных углов.

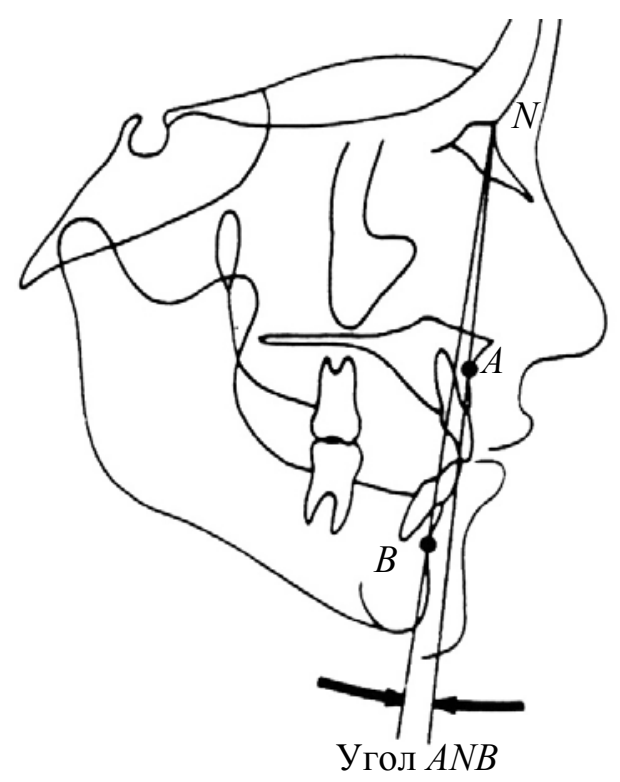

Рис. 3. Угол $A N B$, отвечающий за передне-

заднее положение нижней челюсти относительно верхней (черепа) 


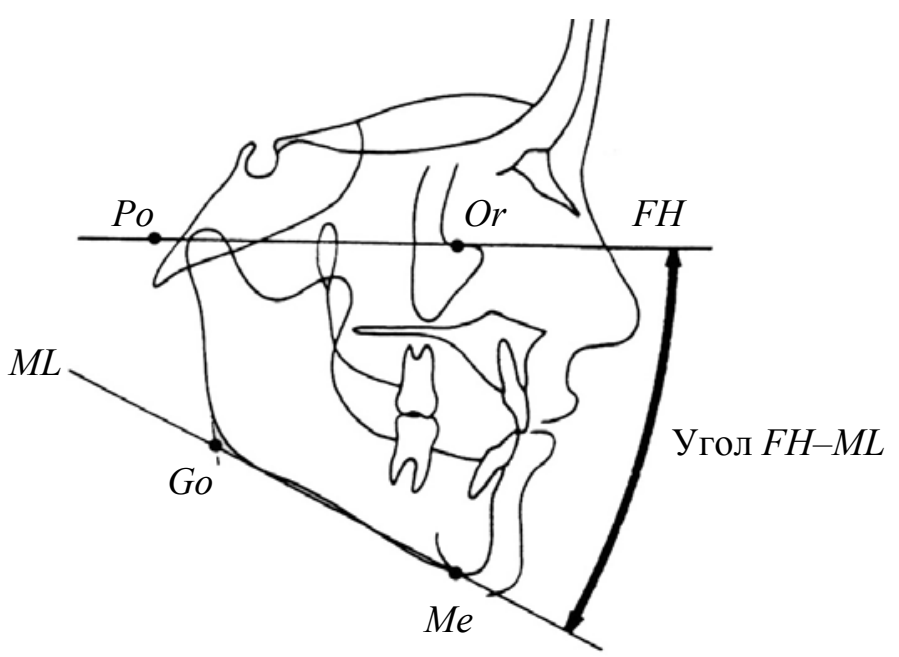

Рис. 4. Угол $F H-M L$, отвечающий за наклон нижней челюсти относительно верхней (черепа)

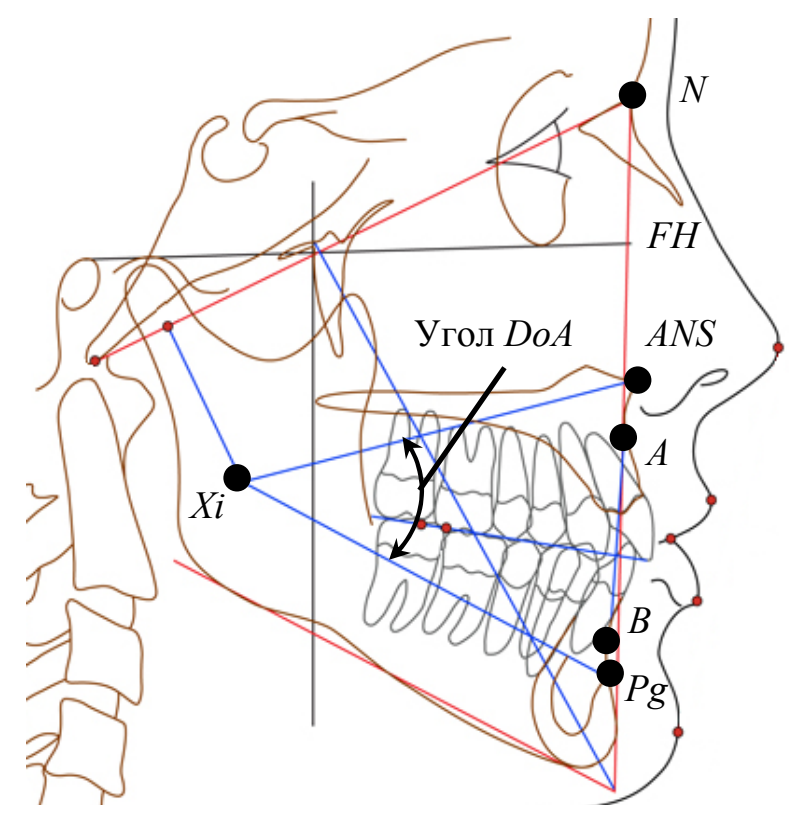

Рис. 5. Угол $D o A$, отвечающий за высоту нижней трети лица

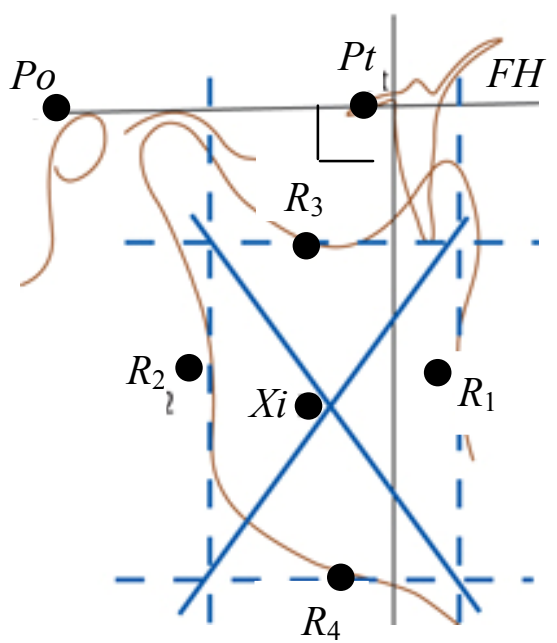

Рис. 6. Определение положения точки $X i$ 
За изменение угла $A N B$ отвечают координаты точек $A, N, B$. Точки $A$ и $N$ принадлежат верхней челюсти и черепу, соответственно, они жестко связаны. Расстояние между точками $A$ и $N$ является фиксированным, и его можно изменить только хирургическим путем. Точка $B$ принадлежит нижней челюсти, и расстояние между ней и точками $A$ и $N$ может варьироваться. Соответственно, изменением только координат точки $B$ можно повлиять на изменение угла $A N B$ при коррекции нехирургическим путем.

За изменение угла $F H-M L$ отвечают координаты точек $\mathrm{Or}, \mathrm{Po}, \mathrm{Me}, \mathrm{Go.}$ Расстояния между двумя точками $M e$ и Go, принадлежащими нижней челюсти и являющимися основой для прямой $M L$, и двумя точками $O r$ и $P o$, принадлежащими черепу и являющимися основой для франкфуртской горизонтали (прямой $F H$ ), являются постоянными.

Изменение угла $D o A$ определяется изменением координат точек $X i$ и $P g$ относительно точки $A N S$ верхней челюсти (положение которой жестко связано с точками $A, N$ и другими точками верхней челюсти), при условии, что расстояние между точками $X i$ и $P g$ является фиксированным (принадлежат нижней челюсти) (см. рис. 5).

\section{ВЫБОР ПЕРЕМЕННЫХ, ОПРЕДЕЛЯЮЩИХ ПОЛОЖЕНИЕ ПРИКУСА}

Движение твердого тела в общем случае описывается системой из шести уравнений. Таким образом, при первоначальной постановке прикуса есть шесть независимых переменных, управляя ими можно привести нижнюю челюсть в нужное положение. В ходе коррекции прикуса стоматолог приводит стоматологические параметры в норму, которая является не набором конкретных значений параметров, а диапазонами их изменения.

Биомеханическое моделирование позволяет выбрать вектор значений, т.е. из диапазона каждого из параметров выбрать конкретное значение. При выборе конкретных значений необходимо учитывать усилия в мышцах, напряжения и деформации, возникающие в мыщелке нижней челюсти и диске височнонижнечелюстного сустава.

Рассмотрим случай центральной окклюзии при выборе прикуса стоматологом для симметричного случая (нижняя челюсть имеет плоскость симметрии $x O z$ ). Будем рассматривать коррекцию прикуса в сагиттальной плоскости. При этом движение нижней челюсти можно считать плоскопараллельным, при нем положение нижней челюсти будет определяться системой из трех уравнений, включающих изменение координат точки $A$ (полюс) и изменение угла поворота ф вокруг полюса:

$$
\left\{\begin{array}{l}
y_{A}=y(t), \\
z_{A}=z(t), \\
\varphi=\varphi(t) .
\end{array}\right.
$$

Через параметры системы (1) можно выразить стоматологические параметры, определяемые при помощи телерентгенографии. Стоматолог на получившемся боковом снимке телерентгенограммы отмечает реперные точки (например, точка $A$ ) и производит вычисления и анализ параметров (например, угол $A N B$ ) (см. рис. 3). Далее, на основании полученных значений параметров выбирается план ортопедического лечения, который заключается в выборе конкретных значений параметров, входящих в диапазоны физиологических значений, и методов достижения этих выбранных величин (применение тех или иных ортопедических конструкций). 
Стоматологи стремятся изменить не только параметры таким образом, чтобы они входили в диапазоны нормальных значений, но и их значения, чтобы они были как можно ближе к средним значениям.

Из анализа вышеупомянутых параметров и литературного обзора методик рассмотрения состояния зубочелюстной системы следует, что в этих работах напрямую не затрагивается состояние и положение диска височно-нижнечелюстного сустава. Наверное, это связано с тем, что рентгеновские методы практически не способны визуализировать мягкотканые и хрящевые структуры. Соответственно, они не могут даже качественно дать информацию о состоянии и патологических процессах в диске височно-нижнечелюстного сустава.

Опосредованно о положении диска височно-нижнечелюстного сустава можно судить по анализу состояния суставной щели височно-нижнечелюстного сустава между суставными поверхностями мыщелка нижней челюсти и суставной ямки и бугорка височной кости. В литературе описаны размеры суставной щели в норме. Компьютерная томография (как наиболее современный рентгеновский метод) позволяет четко увидеть размеры суставной щели, но не позволяет оценить состояние и положение диска в отличие от магнитно-резонансной томографии.

На боковом снимке магнитно-резонансной томографии можно оценить положение и состояние диска височно-нижнечелюстного сустава, а также выделить точку приложения реакции в суставе $[2,17,19,20,25]$. Она находится путем определения кратчайшего расстояния между суставными поверхностями при условии, что принадлежит поверхности мыщелка и диску височно-нижнечелюстного сустава. В этой точке будут прикладываться реакции при определении усилий мышц в процессе смыкания челюстей (рис. 7).

Рассматривая нижнюю челюсть в первом приближении как абсолютно твердое тело, можно сказать, что углы $A N B, F H-M L$ и $D o A$ описывают положение нижней челюсти относительно верхней (черепа), а также положение точки приложения реакции.

Таким образом, за положение нижней челюсти, а также точки приложения реакции по отношению к верхней (черепу) можно выбрать координаты точки $B$ (или $P g$ ) и угол поворота вокруг точки $B$ (или $P g$ ), т.е. три параметра (координаты полюса и угол поворота вокруг полюса, описывающие плоскопараллельное движение нижней челюсти).

Анализ стоматологических параметров, которые были упомянуты выше, позволил понять, что для однозначного описания положения нижней челюсти при плоскопараллельном движении достаточно этих трех параметров.

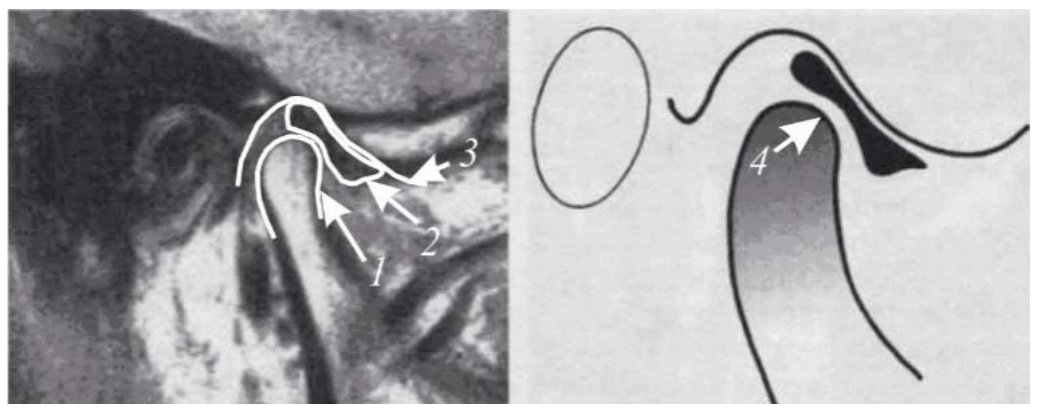

Рис. 7. Магнитно-резонансная томография височно-нижнечелюстного сустава в кососагиттальной проекции (проекция, выполняемая под углом к сагиттальной): 1 - мыщелок; 2 - диск; 3 - суставная ямка и бугорок; 4 - центральная зона диска и вектор жевательной нагрузки, которая противоположна и равна по величине реакции в височно-нижнечелюстном суставе. Нормальное взаиморасположение суставных структур при центральной окклюзии [14] 


\section{ПОСТАНОВКА ЗАДАЧИ БИОМЕХАНИЧЕСКОГО СОПРОВОЖДЕНИЯ КОРРЕКЦИИ ПРИКУСА}

В практике стоматолог в процессе анализа состояния зубочелюстной системы с использованием расчетов на телерентгенограмме отмечает большое количество точек и анализирует множество параметров. Не все выделяемые стоматологом параметры являются независимыми. Многие из них зависят от других и путем применения математических операций выводятся друг из друга.

В данной работе авторы выделили те независимые параметры, которые оказывают влияние и на геометрию, и на напряженно-деформированное состояние зубочелюстной системы.

В качестве параметров, определяющих положение нижней челюсти, были выбраны углы $A N B, D o A$ и $F H-M L$, т.е. $U=\{\angle A N B, \angle D o A, \angle F H-M L\}$. Для каждого из них существуют диапазоны нормальных физиологических диапазонов: $0<\angle A N B<4^{\circ}, 41^{\circ}<\angle D o A<45^{\circ}, 15^{\circ}<\angle F H-M L<25^{\circ}$, со средними (физиологическими) значениями: $\angle A N B_{\text {average }}=2^{\circ}, \angle D o A_{\text {average }}=43^{\circ}, \angle F H-M L_{\text {average }}=20^{\circ}$.

Координаты точки $B$ (или $P g$ ) и угол поворота вокруг этой точки задают положение нижней челюсти относительно верхней (черепа). При изменении координат этой точки и повороте изменяются координаты точек крепления жевательных мышц к нижней челюсти и углы наклонов этих мышц по отношению к франкфуртской горизонтали.

Прикус, определенный стоматологом, задает положение статического равновесия нижней челюсти по отношению к верхней. Это положение челюсти определяется набором параметров $U=\left\{u_{1}, u_{2}, \ldots, u_{m}\right\}$. Физиологическая норма прикуса - диапазоны значений $\breve{u}_{i}<u_{i}<\hat{u}_{i}$, т.е. физиологическая норма представляется $m$-мерным параллелепипедом $\Pi_{m}$. При центральной окклюзии $m=3$, а при отсутствии симметрии $m=6$.

Задача коррекции прикуса состоит в следующем. Когда стоматолог назначает положение прикуса, тогда, согласно изложенному выше, определены конкретные значения $U \in \Pi_{m}$, принадлежащие диапазону физиологической нормы для данного пациента. Обычно повторные обращения пациента к врачу связаны с коррекцией прикуса в этом диапазоне. Поставим задачу коррекции прикуса методами биомеханики.

Будем варьировать значения $U \in \Pi_{m}$ в физиологическом диапазоне. Каждый набор таких параметров с помощью решения статически неопределенной задачи вычисления усилий мышц и реакций височно-нижнечелюстных суставов позволяет найти значение целевой функции, равное $M^{*}$, отражающей напряженность жевательных мышц, $M^{*} \in M=[0,1]$. Задача построения целевой функции рассмотрена ниже. Для каждого набора $U$ имеется точка $M^{*}$, принадлежащая множеству $M$. Тогда будем искать такой набор параметров прикуса $U^{*}$, при котором достигается минимум значений целевой функции $\Phi=\underset{U \in \Pi_{m}}{\min } M^{*}$.

Таким образом, задача биомеханического управления коррекцией прикуса выглядит следующим образом.

Найти

$$
\Phi=\min _{U \in \Pi_{m}} M^{*}
$$


при ограничениях

$$
\begin{gathered}
\sigma_{i}<\sigma_{\text {res }}, \\
\sigma_{i}<\sigma_{\text {col }} .
\end{gathered}
$$

Здесь $\sigma_{i}$ - интенсивность касательных напряжений; $\sigma_{\text {res }}-$ максимальная интенсивность напряжений, при которых не наблюдается резорбция костной ткани в мыщелке нижней челюсти; $\sigma_{c o l}-$ максимальная допустимая интенсивность напряжений в диске височно-нижнечелюстного сустава. Для выполнения ограничений (3)-(4) требуется при построении множества $M$ решение задач теории упругости для определения напряженно-деформированного состояния диска височнонижнечелюстного сустава и нижней челюсти. При различных значениях $U \in \Pi_{m}$ будут получены различные усилия мышц и реакции сустава. Критерий (2) устроен таким образом, что позволяет избежать перенапряжения мышц зубочелюстной системы, доступных и не доступных при пальпации стоматологом, что может приводить к болевым ощущениям, перегрузке диска, неправильному процессу открывания и закрывания рта.

При заданном положении прикуса определим величины усилий мышц и реакцию в височно-нижнечелюстном суставе с помощью раскрытия статической неопределенности при использовании критерия оптимизации. Его выбор обсуждается в работах [3, 9, 22, 24].

При заданном положении прикуса имеет место статическое равновесие нижней челюсти. Условия статического равновесия можно записать в виде уравнений (5) и (6):

$$
\begin{gathered}
\sum_{i=1}^{N} \mathbf{F}_{i}+\sum_{j=1}^{2} \mathbf{R}_{j}+\mathbf{F}_{b}=0 \\
\sum_{i=1}^{N} \mathbf{M}_{i}+\sum_{j=1}^{2} \mathbf{M}_{j}+\mathbf{M}_{b}=0 .
\end{gathered}
$$

Здесь $N$ - число рассматриваемых мышц; $\mathbf{F}_{i}$ - усилия жевательных мышц; $\mathbf{R}_{j}$ - реакции височно-нижнечелюстных суставов; $\mathbf{F}_{b}$ - сила сжатия челюстей; $\mathbf{M}_{i}, \quad \mathbf{M}_{j}, \quad \mathbf{M}_{b}$ - моменты усилий жевательных мышц, реакций височнонижнечелюстных суставов и силы сжатия челюстей относительно неподвижной точки $O$. Необходимо отметить, что поскольку направление суставных реакций височно-нижнечелюстных суставов заранее неизвестно, то реакция в каждом суставе представляется в виде трёх взаимно перпендикулярных базисных компонентов.

Идея введения данного критерия состоит в следующем. В случае, когда жевательная система развивает определённую по направлению (желаемую) силу сжатия, количество возможных вариантов вовлечения жевательных мышц в процесс сжатия ограничивается. Когда величина силы сжатия увеличивается, область возможных вариантов вовлечения каждой мышцы в создание данной силы уменьшается. Уменьшение происходит из-за неспособности отдельных мышечных элементов производить силы, которые превышают максимальную мышечную силу данного элемента. Когда достигается максимальная сила сжатия челюстей, ограниченная область мышечных вовлечений сокращается до единственного варианта. Похожую ситуацию можно увидеть, когда имеется система двух идеально-пластичных параллельных жестко связанных стержней, нагруженных силой. При этом у одного из стержней предел текучести меньше, чем у другого. Очевидно, что при достижении 
наименьшего предела текучести для стержней вся несущая способность осуществляется вторым стержнем, имеющим больший предел текучести.

Определим вектор значений мышечных сил $\mathbf{X}=\left\{F_{1}, F_{2}, \ldots, F_{N}\right\}$ и функции $f_{i}(\mathbf{X})=\frac{F_{i}}{F_{i, \max }}$, где $i \in[1,2, \ldots, N]$ и $F_{i, \max }$ - максимальная мышечная сила $i$-й мышцы, которая определяется как

$$
F_{i, \max }=K A_{i} .
$$

Здесь $K$ - удельная мышечная сила, определенная экспериментально для того типа мышц, к которому относятся мышцы зубочелюстной системы, $K=37 \mathrm{H} \cdot \mathrm{cm}^{-2}[15] ; A_{i}$ - физиологическое поперечное сечение $i$-й мышцы.

Определим множество $\Omega$, образованное теми значениями $\mathbf{X}$, при которых выполняются уравнения равновесия (5) и (6). Тогда требуется найти точку $\mathbf{X}^{*} \in \Omega$ (значения усилий мышц), для которой

$$
\max _{i \in[1,2, \ldots, N]} f_{i}\left(\mathbf{X}^{*}\right)=\min _{\mathbf{X} \in \Omega} \max _{i \in[1,2, \ldots, N]} f_{i}(\mathbf{X}) .
$$

Таким образом, точка $\mathbf{X}^{*}$, определенная критерием (8), разыскивается как минимум среди максимальных значений отношений $\frac{F_{i}}{F_{i, \max }}$, что обеспечивает наиболее равномерное вовлечение в действие всей группы мышц зубочелюстной системы.

Из вида целевой функции следует, что необходимо осуществлять поиск минимума среди континуума максимальных значений (задача на минимакс). Целевая функция для данной задачи является дискретной функцией номеров мышц. Но данная дискретная функция может быть преобразована в линейную целевую функцию (9) с $N$ дополнительными линейными ограничениями (10) и (11).

Введем функцию $\mu(\mathbf{X})=\max _{i \in[1,2, \ldots, N]} f_{i}(\mathbf{X})$. Очевидно, значение максимума принадлежит ограниченной области $0 \leq \mu \leq 1$. Тогда будем решать следующую задачу.

Найти такой вектор усилий $\mathbf{X}^{*}$, при котором достигается

$$
\min _{\mathbf{X} \in \Omega} \mu(\mathbf{X})
$$

при ограничениях

$$
\begin{gathered}
f_{i}(\mathbf{X}) \leq \mu, \\
0 \leq \mu \leq 1,
\end{gathered}
$$

где $i \in[1,2, \ldots, N]$.

Для каждого найденного вектора усилий $\mathbf{X}^{*}$ значение целевой функции (9) определяет величину $M^{*}$, т.е. $M^{*}=\min _{\mathbf{X} \in \Omega} \mu(\mathbf{X})$. При изменении управляющих параметров $U$ строится множество $M$, что и позволяет в дальнейшем найти $\Phi=\min _{U \in \Pi_{m}} M^{*}$ и тем самым решить задачу управления (2)-(4).

Таким образом, от первоначальной дискретной задачи оптимизации с шестью ограничениями осуществляется переход к линейной задаче оптимизации с целевой функцией (9), в которой целевая функция $\mu(\mathbf{X})$ должна быть минимизирована, удовлетворяя условиям $N+6$ линейных ограничений уравнений (5), (6) и (10), (11) с двумя дополнительными условиями. 
1. Мышечная сила должна быть больше нуля или равняться нулю. Данное ограничение обусловлено следующим обстоятельством: с точки зрения механики сухожильно-мышечные комплексы следует рассматривать как односторонние связи, так как они способны сопротивляться растяжению и, подобно гибким нитям, выключаются из работы при появлении в них сил осевого сжатия.

2. Можно использовать экспериментальные данные о том, что суставные реакции по оси $x$ должны действовать в заднем направлении; суставные реакции по оси $z$ должны действовать в нижнем направлении [14, 23, 24]. Данное ограничение может быть использовано, потому что при сжатии наибольшее число мышц действует вперед и вверх, таким образом суставная реакция должна противодействовать мышечным силам, чтобы поддерживать статическое равновесие. Это ограничение не является обязательным.

Целевая функция (9) ограничена сверху, а ограничения (5), (6) и (10) линейны. Так как переменные задачи заданы на пересечении замкнутого отрезка (ограничение для величины $\mu$ ), плоскости (для реакций) и полуплоскости (для величины усилий в мышцах) (выпуклые множества), то ввиду линейности ограничений, а значит их непрерывности, область, заданная ограничениями, является выпуклой и замкнутой. Такая задача является классической задачей линейного программирования и имеет единственное решение, поэтому она может быть решена симплекс-методом для линейного программирования.

Окончательно решаемая задача может быть сформулирована следующим образом:

найти

$$
\min _{\mathbf{X} \in \Omega} \mu(\mathbf{X})
$$

при ограничениях

$$
\begin{gathered}
\sum_{i=1}^{N} F_{i x}+\sum_{j=1}^{2} R_{j x}+F_{b x}=0, \\
\sum_{i=1}^{N} F_{i y}+\sum_{j=1}^{2} R_{j y}+F_{b y}=0, \\
\sum_{i=1}^{N} F_{i z}+\sum_{j=1}^{2} R_{j z}+F_{b z}=0, \\
\sum_{i=1}^{N} M_{i x}+\sum_{j=1}^{2} M_{j x}+M_{b x}=0, \\
\sum_{i=1}^{N} M_{i y}+\sum_{j=1}^{2} M_{j y}+M_{b y}=0, \\
\sum_{i=1}^{N} M_{i z}+\sum_{j=1}^{2} M_{j z}+M_{b z}=0, \\
0 \leq \mu \leq 1, \\
\frac{F_{i} \geq 0,}{F_{i, \max }} \leq \mu,
\end{gathered}
$$


где $F_{i}$ - усилие, развиваемое $i$-й мышцей $(i=1, \ldots, N) ; R_{j}$ - реакция височнонижнечелюстного сустава $(j=1,2) ; \quad F_{b}$ - сила сжатия челюстей; $F_{i, \max }$ максимальное усилие, развиваемое $i$-й мышцей.

В результате решения находятся мышечные усилия и реакции в суставах.

В итоге решаются две связанные задачи: определение усилий мышц и реакций в височно-нижнечелюстных суставах в процессе смыкания челюстей (приложена сила сжатия) и определение напряженно-деформированного состояния нижней челюсти и диска височно-нижнечелюстного сустава при двух фиксированных значениях параметров и при изменении третьего (например, величины углов $D o A$ и $F H-M L$ фиксируются и меняется величина угла $A N B$ в диапазоне нормальных значений). Далее фиксируются величины углов $A N B$ и $D o A$ и меняется величина угла $F H-M L$. В последнем случае фиксируются величины углов $A N B$ и $F H-M L$ и меняется величина угла $D o A$. Набор этих углов составляет вектор управляющих параметров $U=\left\{u_{1}, u_{2}, u_{3}\right\}$, по которым ищется минимум целевой функции (задачи (2)-(4)).

В каждом случае для каждого значения варьируемого параметра определяется точка приложения реакции височно-нижнечелюстного сустава, находятся усилия мышц и реакция височно-нижнечелюстного сустава, вычисляются напряжения в нижней челюсти и диске височно-нижнечелюстного сустава, а также сравниваются с предельными значениями. Важно также, что определяется значение $\mu(\mathbf{X})=\max _{i \in[1,2, \ldots, N]} f_{i}(\mathbf{X})$, где $\mathbf{X}=\left\{F_{1}, F_{2}, \ldots, F_{N}\right\}$, функция $f_{i}(\mathbf{X})=\frac{F_{i}}{F_{i, \max }}, i \in[1,2, \ldots, N]$ и $F_{i, \max }$ - максимальная мышечная сила $i$-й мышцы.

При решении всех задач для вышеупомянутых случаев фиксируемых и варьируемых параметров определяется множество значений $\mu$, т.е. $\mu=\mu(\angle A N B, \angle D o A, \angle F H-M L)$. Каждой точке с координатами $(\angle A N B, \angle D o A, \angle F H-M L)$ соответствует некоторое значение $\mu$, являющееся элементом множества $M(11)$. Нас интересует минимальное значение $\mu$ при условии, что напряжения при этом $\mu=M^{*}$ не превышают заданных пределов (задача (2)-(4)).

Окончательно решение последовательности поставленных задач составляет методику биомеханической коррекции прикуса, результатом применения которой является объективизированный набор параметров, определяющих прикус. Это позволяет ввести в практику стоматологии количественные соотношения, что должно привести к уменьшению числа повторных посещений стоматолога. На рис. 8 представлена схема применения методики [6].

Таким образом, поставленная задача управления коррекцией прикуса заключается в следующем. У заданного стоматологом положения прикуса имеется физиологический диапазон, в котором меняются управляющие параметры $U$. Для каждого такого набора решается задача определения усилий мышц и реакций в суставах (задача (12), (13)). Для каждого решения имеются различные значения целевой функции (12), которые позволяют построить множество $M$ и решить задачу (управления (2)-(4)). 


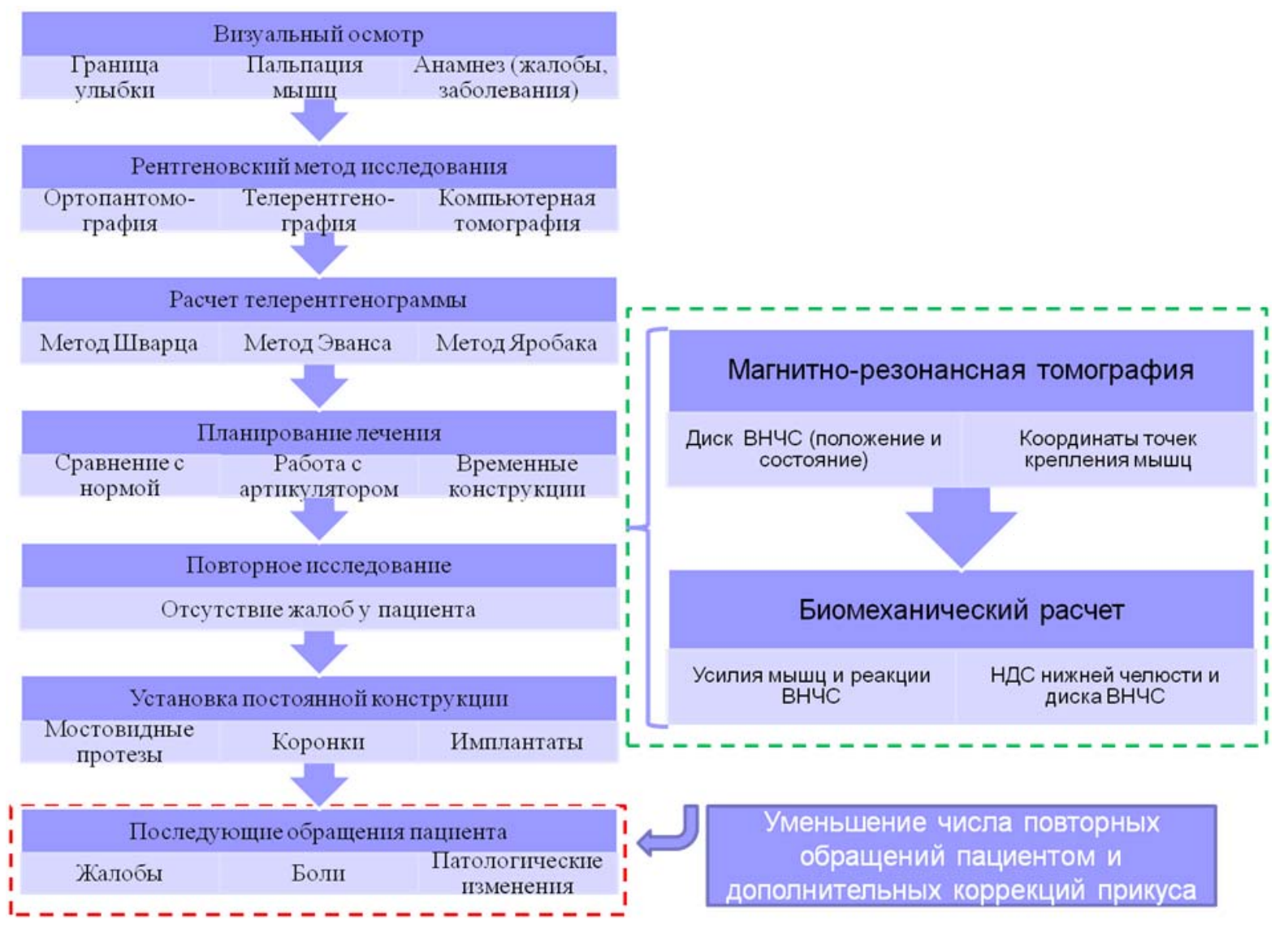

Рис. 8. Методика биомеханического сопровождения коррекции прикуса: ВНЧС височно-нижнечелюстной сустав, НДС - напряженно-деформированное состояние

\section{ЗАКЛЮЧЕНИЕ}

Возможно, стоматолог выскажет мнение, что на практике сложно достичь значений углов $A N B, D o A$ и $F H-M L$, которые рекомендуются авторами для данного пациента, тогда он сможет рассмотреть те случаи, в которых значения близки к рекомендуемому на основании биомеханического моделирования. Реализовать их можно, применив те или иные ортопедические конструкции.

Полученные результаты позволяют дать объективные количественные оценки усилий, возникающих в нижней челюсти и диске височно-нижнечелюстного сустава для конкретного пациента, и дать долговременный прогноз возникновения патологических процессов, связанных с коррекцией прикуса.

\section{БЛАГОДАРНОСТИ}

Работа выполнена при финансовой поддержке РФФИ (грант № 15-01-04884-a, «Биомеханическое моделирование формирования и развития трабекулярной костной ткани в различных отделах скелета человека в норме и при патологии»).

\section{СПИСОК ЛИТЕРАТУРЫ}

1. Анатомия и биомеханика зубочелюстной системы / под ред. Л.Л. Колесникова, С.Д. Арутюнова, И.Ю. Лебеденко. - М.: Практическая медицина, 2007. - 224 с.

2. Аун М., Менар М., Морлье Ж., Рамос А., Монеде-Хокуард Л., Сид М. Разработка и проверка двумерной конечно-элементной модели височно-нижнечелюстного сустава при помощи магнитнорезонансного исследования: моделирование движения открытия и закрытия челюстей // Российский журнал биомеханики. - 2011. - Т. 15, № 1. - С. 23-32. 
3. Зациорский В.М., Прилуцкий Б.И. Нахождение усилий мышц человека по заданному движению // Современные проблемы биомеханики. - 1992. - Вып. 7. - С. 81-123.

4. Карманов В.Г. Математическое программирование. - М.: Наука, 1975. - 272 с.

5. Киченко А.А., Шумихин А.Ю., Тверье В.М., Няшин Ю.И., Симановская Е.Ю., Еловикова А.Н. Определение усилий, возникающих в жевательной системе человека // Российский журнал биомеханики. - 2004. - Т. 8, № 4. - С. 27-38.

6. Никитин В.Н. Методика коррекции прикуса зубочелюстной системы человека на основе биомеханического моделирования // Материалы XI Всероссийского съезда по фундаментальным проблемам теоретической и прикладной механики, 20-24 августа 2015. - Казань, 2015. - С. 27752777.

7. Няшин Ю.И., Тверье В.М., Лохов В.А., Менар М. Височно-нижнечелюстной сустав человека как элемент зубочелюстной системы: биомеханический анализ // Российский журнал биомеханики. 2009. - T. 13, № 4. - С. 7-21.

8. Тверье В.М. Биомеханическое моделирование онтогенеза зубочелюстной системы человека // Материалы XI Всероссийского съезда по фундаментальным проблемам теоретической и прикладной механики, 20-24 августа 2015. - Казань, 2015. - С. 3684-3686.

9. Тверье В.М., Няшин Ю.И., Никитин В.Н. Биомеханическая модель определения усилий мышц и связок в зубочелюстной системе человека // Российский журнал биомеханики. - 2013. - Т. 17, № 2. - С. 8-20.

10. Тверье В.М., Няшин Ю.И., Никитин В.Н., Оборин Л.Ф. Механическое давление как основа биомеханического моделирования зубочелюстной системы человека // Российский журнал биомеханики. - 2014. - Т. 18, № 1. - С. 24-35.

11. Тверье В.М., Симановская Е.Ю., Няшин Ю.И. Атрофический синдром, связанный с изменениями биомеханического давления в зубочелюстной системе человека // Российский журнал биомеханики. 2006. - T. 10, № 1. - С. 9-14.

12. Тверье В.М., Симановская Е.Ю., Няшин Ю.И. Биомеханическое давление, сопутствующее формированию зубоальвеолярного блока у человека // Российский журнал биомеханики. - 2005. Т. 9, № 3. - С. 9-15.

13. Тверье В.М., Симановская Е.Ю., Еловикова А.Н., Няшин Ю.И., Киченко А.А. Биомеханический анализ развития и функционирования зубочелюстной системы человека // Российский журнал биомеханики. - 2007. - Т. 11, № 4. - С. 84-104.

14. Хватова В.А. Клиническая гнатология. - М.: Медицина, 2005. - 296 с.

15. Barbenel J.C. The biomechanics of temporomandibular joint: a theoretical study // Journal of Biomechanics. - 1972. - Vol. 5, № 3. - P. 251-256.

16. Gröning F., Fagan M., O'Higgins P. Modeling the human mandible under masticatory loads: which input variables are important? // Anat. Rec. (Hoboken). - 2012. - Vol. 295, № 5. - P. 853-863. DOI: 10.1002/ar.22455.

17. Hyoun-Suk Ahn, Su-Beom Cho, Kwang-Joon Koh. Positional and morphologic changes of the temporomandibular joint disc using magnetic resonance imaging // Korean Journal of Oral and Maxillofacial Radiology. - 2001. - Vol. 31. - P. 235-240.

18. Ingawale S.M., Goswami T. Biomechanics of the temporomandibular joint // Human Musculoskeletal Biomechanics / ed. by T. Goswami. - Rijeka, 2012. - 244 p.

19. Iwasaki L.R., Crosby M.J., Gonzalez Y., McCall W.D., Marx D.B., Ohrbach R., Nickel J.C. Temporomandibular joint loads in subjects with and without disc displacement // Orthopedic Reviews. 2009. - Vol. 1. - P. 90-93.

20. Manfredini D., Basso D., Arboretti R., Guarda-Nardini L. Association between magnetic resonance signs of temporomandibular joint effusion and disk displacement // Oral. Surg. Oral. Med. Oral. Pathol. Oral. Radiol. Endod. - 2009. - Vol. 107. - P. 266-271.

21. Nordin M., Franke V.H. Basic Biomechanics of the Musculoskeletal System. - 3rd ed. - Philadelphia: Lippincott Williams \& Wilkins, 2001. - 496 p.

22. Osborn J.W. Features of human jaw design which maximize the bite force // Journal of Biomechanics. 1996. - Vol. 29, № 5. - P. 589-595.

23. Osborn J.W., Baragar F.A. Predicted pattern of human muscle activity during clenching derived from a computer assisted model: symmetric vertical bite forces // Journal of Biomechanics. - 1985. - Vol. 18, № 8. - P. 599-612.

24. Pedotti A., Krishman V.V., Stark L. Optimization of muscle-force sequencing in human locomotion // Mathematical Biosciences. - 1978. - Vol. 38, № 1/2. - P. 57-76.

25. Pérez del Palomar A., Doblaré M. An accurate simulation model of anteriorly displaced TMJ discs with and without reduction // Med. Eng. Phys. - 2007. - Vol. 29, № 2. - P. 216-226. 
26. Throckmorton G.S. Quantitative calculations of temporomandibular joint reaction forces. Part II. The importance of the direction of the jaw muscle forces // J. Biomech. - 1985. - Vol. 18, № 6. - P. 453-461.

\section{THE PROBLEM OF OCCLUSION CORRECTION IN THE HUMAN MAXILLOFACIAL SYSTEM}

\section{V.M. Tverier, V.N. Nikitin (Perm, Russia)}

Dentists rely on their experience or use techniques, which are related to geometric calculations at correction of occlusion associated with the loss of teeth, increased dental abrasion, injuries, and fractures of jaws. Occlusion determines the loading of the mandible and the temporomandibular joint, so biomechanical modelling is needed for correction. The problem of occlusion correction control is formulated. Stresses into the temporomandibular disc and the mandible should not exceed the specified limits for the intensity of the stresses at parameters determining occlusion state. Stresses and strains into the body of the mandible and disc are determined by solving the two problems of theory of elasticity: for the mandible and the disc in the central occlusion at maximal loaded muscles. The application point of the articular reaction and the disc position are determined by results of magnetic resonance imaging. It is necessary to know muscle efforts to determine the stress-strain states of the jaws and the temporomandibular joint disc. Muscle efforts and the reaction are boundary conditions for these problems. Muscle efforts are beforehand unknown. Muscle efforts are determined by solving the optimization problem, which includes static equations of the mandible at the action of given masticatory load and optimization criterion. The initial problem of determining the stress-strain state of the mandible and the disc is solved after calculating muscle efforts and the temporomandibular joint reaction. Values of parameters determining the position of the occlusion are calculated taking into account the comparison of the stresses with the permissible stresses.

Key words: occlusion correction, maxillofacial system, temporomandibular joint, muscle efforts, articular disc. 